\title{
PLANIFICACIÓN DEL ENTRENAMIENTO DEPORTIVO EN JUEGOS ESCOLARES CENTROAMERICANOS Y DEL CARIBE ARMENIA 2013
}

\begin{abstract}
Ramos.S, 1, Ayala. C 2 Garcia.M ${ }^{3}$
Santiago Ramos B: Licenciado en educación física y deporte. Magister en metodología del entrenamiento deportivo con mención en atletismo. Profesor titular Universidad de Caldas, Manizales, Colombia. Grupo Cumanday actividad física y deporte. santiago.ramos@ucaldas.edu.co

Carlos Federico Ayala Z: Licenciado en educación física, recreación y deporte. Especialista en Procesos Pedagógicos del Entrenamiento Deportivo. Magister en Educación. Doctor en ciencias de la motricidad humana. Profesor asistente de la Universidad de Caldas, Manizales, Colombia. Grupo Cumanday actividad física y deporte. federico.ayala@ucaldas.edu.co
\end{abstract}

Angélica María García G: Licenciada en educación física, recreación y deporte. Magister en intervención integral en el deportista. Profesora de la Universidad de Caldas, Manizales, Colombia. Grupo Cumanday actividad física y deporte. angelica.garcia@ucaldas.edu.co

\section{RESUMEN}

Con el objetivo de conocer las características de la planificación del entrenamiento que emplearon entrenadores participantes en los Juegos Escolares Centroamericanos y del Caribe, realizados en Armenia, Colombia entre octubre y noviembre de 2013, se realizó un estudio cuantitativo, prospectivo, observacional, con alcance descriptivo y relacional, de corte transversal, en el cual fue aplicada una encuesta elaborada por el grupo investigador a una muestra no probabilística de 41 entrenadores (35 hombres, 6 mujeres), de 13 modalidades deportivas, integrantes de las nueve

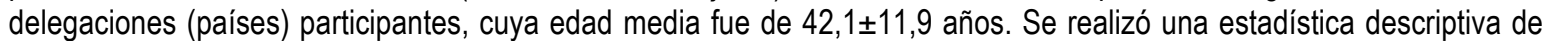
análisis de frecuencias, medidas de tendencia central y relaciones con el programa SPSS v. 20. Los resultados en cuanto al tiempo al cual planificaban muestran, en primer lugar, el largo plazo; acerca del criterio para determinar el modelo de planificación a seguir, la mayoría utilizó todos los criterios propuestos (objetivos, calendario y deportistas); en cuanto a si utilizaban alguno de los modelos presentes en la literatura, predominó la respuesta afirmativa, siendo los macrociclos los más utilizados. En conclusión el tiempo de planificación empleado fue preferentemente a largo plazo, el criterio para determinar el modelo de planificación a seguir, en su orden fueron todos los criterios, los objetivos competitivos y el calendario de competencia, la gran mayoría tienen conocimiento y utilizan alguno de los modelos de planificación conocidos en la literatura especializada.

PALABRAS CLAVE Entrenamiento físico, rendimiento deportivo, planificación.

\begin{abstract}
In order to know the characteristics of planning training employed by coaches participating in the Central American and Caribbean School Games, held in Armenia, Colombia between October and November 2013, a quantitative, prospective, observational study was conducted with descriptive and relational, cross-sectional scope, it was applied a survey conducted by the research group to a nonrandom sample of 41 coaches (35 men, 6 women) of 13 different sports, members of the nine delegations (countries) participants, whose mean age was $42.1 \pm 11.9$ years. Descriptive statistics of frequency analysis, measures of central tendency and relations with the SPSS program $\vee 20$ was performed. The results regarding the time at which planned show, first, the long term; about the criteria to determine the planning model to follow, most it used all (objectives, calendar and athletes) proposed criteria; as to whether any of the models used in the literature, the prevailing yes, being the most used macrocycles. In conclusion planning time employee was preferably long term, the criterion for determining the planning model to follow, in their order were all criteria, competitive objectives and competition schedule, the vast majority have knowledge and use any of planning models known in the literature.
\end{abstract}

KEY WORDS Physical training, sports performance, planning,

Convocatoria ii trimestre. Recibido el 1 de Marzo de 2017; Aceptado el 1 de junio de 2017. 


\section{INTRODUCCION}

El entrenamiento deportivo, en su transcurso por la historia se ha caracterizado por constantes cambios, especialmente a partir de la década del 50 del siglo pasado, lo que ha influido en que actualmente esté marcado u orientado por procesos metodológicos determinados sustancialmente por la cientificidad (Fernández, 1981) (Issurin, 2012) (Navarro, Oca, \& Rivas, 2010), por el elevado nivel de rendimiento, extensos calendarios de eventos competitivos programados en el transcurso del año en los diferentes deportes y alto grado de especialización deportiva que se requiere para el alto rendimiento (Bompa, 2002) (Filin, 1996) (Ramos, Ayala, \& Aguirre, 2012) (Verjoshansky, 2001). Frente a estas realidades, los procesos de planificación deportiva, acentúan sus desarrollos en los aspectos cuantitativos y cualitativos (Campos \& Cervera, 2003) de la preparación técnica, táctica, física, psicológica y teórica, con el fin de que los deportistas alcancen rendimientos elevados durante su proceso competitivo.

Es por eso que la Teoría y Metodología del Entrenamiento Deportivo, propende por una planificación centrada en el mejoramiento y mantenimiento de las capacidades condicionales, coordinativas y funcionales, teniendo en cuenta diferentes direccionamientos de tipo científico, administrativo, fisiológico, educativo y de orientaciones profesionales, con el fin de alcanzar los objetivos planeados mediante etapas como el conocer, organizar, observar, analizar, planear, adaptar, aplicar y tomar decisiones (Ayala, Alzate, \& Aguirre, 2014) (Fernández, 1981) (Roberton \& Halverson, 1984); lo anterior es necesario para orientar el trabajo con seguridad, tanto para el deportista como para el proceso y, así atender y alcanzar los principios y objetivos propuestos (Filin, 1996) (Gomes, 2009) (Machado, 2011) (Ramos, Ayala, \& Aguirre, 2012).

Es por ello que se entiende la necesidad de que los entrenadores sean personas idóneas y con un amplio conocimiento de los diferentes aspectos inherentes a la formación y preparación del deportista. Aspectos de la planificación tales como los objetivos deportivos, las competiciones, la previsión y control del entrenamiento, la organización de las cargas, la periodicidad (macro, meso y microciclos) y ciclicidad (corto, mediano y largo plazo) del entrenamiento, en función del nivel del deportista, son necesarios para orientar la preparación del deportista de manera planificada, estratégica y en construcción progresiva, para el adecuado desarrollo de la forma deportiva (Barbanti, 1979) (Campos \& Cervera, 2003) (Fernández, 1981) (Filin, 1996) (García \& Navarro, 1996) (Gómez, 2011) (Issurin, 2012).

Con base en lo anterior y el proceso de investigación llevado a cabo con entrenadores Centro-Americanos y del Caribe, en los Juegos Escolares Armenia 2013 y estos como orientadores de deportistas jóvenes, se llama la atención sobre el procedimiento de la planificación en la vida deportiva de sus alumnos deportistas. Siendo que, cuando se planifica el proceso de adaptación y preparación deportiva, se debe hacer con fundamento en objetivos y contenidos del entrenamiento, en las fases sensibles del desarrollo de las capacidades coordinativas y condicionales, ya que, el proceso de entrenamiento en estas edades se debe centrar esencialmente en una preparación básica y variada de las mismas (Barbanti, 1979) (Filin, 1996) (Martin, Nicolaus, Ostrowski, \& Rost, 2004), porque se necesita planificar el proceso de entrenamiento a partir de los niveles, objetivos y ritmos de aprendizaje del deportista joven, propendiendo por la individualidad y no la estandarización, asumir sus satisfacciones y necesidades frente al deporte y el fomento de la ética deportiva (Murcia, Taborda, \& Ángel, 1998).

La planificación deportiva, también es un proceso educativo de muchos años (plan perspectivo), orientado hacia el alto rendimiento, donde se deben tener en cuenta la edad y preparación del deportista, sus particularidades, su forma de vida, sus puntos flacos y fuertes (Fernández, 1981) (Filin, 1996) (Matveyev, 1985), para plantear la iniciación, fundamentación y perfeccionamiento deportivo (formación) multivariado, en los contextos educativos formales y no formales (Platonov, 2001) o como complemento de las clases de educación física para el mejoramiento de la salud, el interés por la actividad física y el ejercicio, y la motivación por la práctica de deportes (Rusch \& Weineck, 2004), o sea, el entrenamiento es el común denominador y el deportista el protagonista principal del proceso de preparación y formación deportiva. 
En la etapa inicial de preparación y formación deportiva, es adecuado tener como objetivos principales el aprendizaje y perfeccionamiento de la técnica deportiva, asegurar el entrenamiento de manera individual y desarrollar las diferentes capacidades y habilidades que son indispensables para la continuación del proceso (Gomes, 1998).

Si bien se han mostrado ventajas significativas del modelo de bloques sobre el tradicional en piragüistas de nivel mundial (García-Pallares, García-Fernández, Sánchez-Medina, \& Izquiertdo, 2015), encontrando que los bloques lograron resultados similares con la mitad del entrenamiento del modelo tradicional, hay consenso que en los jóvenes, se recomienda el modelo tradicional.

Para al análisis, los deportes fueron agrupados siguiendo la propuesta de deportes de resistencia, de fuerza velocidad, de pelota, de combate y de precisión y arte competitivo (Lanier, 1997).

Por todo lo anterior se hizo necesario estudiar algunos aspectos relacionados con la planificación del entrenamiento deportivo en el contexto escolar Centro-Americano y del Caribe tales como el tiempo al cual planifican, los criterios utilizados para determinar el modelo de planificación y si se utilizaba alguno de los modelos de planificación de la literatura.

\section{MODELO METODOLOGICO}

\section{MÉTODOS}

El estudio dentro del enfoque cuantitativo, observacional, prospectivo, tuvo alcance descriptivo y correlacional con corte transversal. El total de entrenadores participantes en los Juegos no pudo ser determinado, pues aparecían mezclados con utileros, personal médico, delegados y otras personas en la categoría "oficiales", por tanto la muestra no fue probabilística. Fueron encuestados 41 entrenadores deportivos (35 hombres, 6 mujeres) de los nueve países participantes. La técnica utilizada fue la encuesta, elaborada por el grupo investigador, sometida a criterio de expertos nacionales y ya utilizada en 2010 con ocasión de los Juegos Suramericanos Medellín 2010, que constaba de 24 preguntas que indagan acerca del tiempo de planificación más empleado, el criterio utilizado para determinar el modelo de planificación a seguir, si seguían alguno de los modelos de planificación conocidos, entre otros.

El procedimiento consistió en abordar a los entrenadores a la salida de los escenarios deportivos, y después de explicarles las características del estudio, aplicarles la encuesta, previa garantía del anonimato, que sus respuestas serían utilizadas exclusivamente con fines científicos y académicos y que podían no participar o no contestar alguna de las preguntas sin que esto tuviera consecuencia alguna para ellos 0 sus delegaciones. Fueron entrenados estudiantes de último año de educación física para la aplicación de las encuestas.

El tratamiento de los resultados consistió en análisis de medidas de tendencia central (media) y dispersión (desviación estándar), frecuencias y relación entre variables cualitativas mediante la correlación por rangos (Rho) de Spearman y entre las cuantitativas mediante el coeficiente de correlación de Pearson, con un $\alpha=0.05$, con el programa SPSS versión 20.

\section{RESULTADOS}

La tabla 1 presenta las características sociodemográficas de la muestra evaluada, donde resalta la baja participación femenina, común en la mayoría de deportes, anotándose que de las 6 encuestadas, 5 tienen formación universitaria y 1 de postgrado, y que de ellas 4 son entrenadoras de deportes de fuerza-velocidad, 1 de deporte de combate y 1 de pelota.
Tabla
1.
Características Sociodemográficas de la población 
evaluada (promedio $X$, desviación estándar $D E$, número $N$ y porcentajes \%).

\begin{tabular}{|c|c|c|c|c|c|c|}
\hline \multirow[t]{2}{*}{$\begin{array}{l}\text { Característ } \\
\text { ica }\end{array}$} & \multicolumn{2}{|c|}{ Todos } & \multicolumn{2}{|c|}{$\begin{array}{r}\text { Masculin } \\
\text { o }\end{array}$} & \multicolumn{2}{|c|}{$\begin{array}{r}\text { Femenin } \\
\text { o }\end{array}$} \\
\hline & $\mathrm{X}$ & $\begin{array}{l}\mathrm{D} \\
\mathrm{E}\end{array}$ & $\mathrm{X}$ & $\mathrm{DE}$ & $\mathrm{X}$ & $\begin{array}{l}\mathrm{D} \\
\mathrm{E}\end{array}$ \\
\hline Edad (años) & $\begin{array}{r}42.0 \\
8\end{array}$ & $\begin{array}{r}11 \\
.8 \\
5\end{array}$ & $\begin{array}{r}42 \\
.6 \\
2 \\
\end{array}$ & $\begin{array}{r}12.4 \\
1\end{array}$ & 39.0 & $\begin{array}{r}8 . \\
1\end{array}$ \\
\hline & $\mathrm{N}$ & $\%$ & $\mathrm{~N}$ & $\%$ & $\mathrm{~N}$ & $\%$ \\
\hline Sexo & 41 & $\begin{array}{r}10 \\
0\end{array}$ & 35 & 85.4 & 6 & $\begin{array}{c}1 \\
4 . \\
6\end{array}$ \\
\hline \multicolumn{7}{|c|}{ Escolaridad (máximo nivel aprobado) } \\
\hline Técnico & 4 & $\begin{array}{c}9 . \\
76\end{array}$ & 4 & $\begin{array}{r}11.4 \\
3\end{array}$ & 0 & 0 \\
\hline Tecnólogo & 2 & $\begin{array}{r}4 . \\
87\end{array}$ & 2 & 5.71 & 0 & 0 \\
\hline $\begin{array}{r}\text { Universitari } \\
0\end{array}$ & 23 & $\begin{array}{r}56 \\
.0 \\
9\end{array}$ & 18 & $\begin{array}{r}51.4 \\
3\end{array}$ & 5 & $\begin{array}{c}8 \\
3 . \\
3 \\
3\end{array}$ \\
\hline Postgrado & 11 & $\begin{array}{r}26 \\
.8 \\
4\end{array}$ & 10 & $\begin{array}{r}28.5 \\
7\end{array}$ & 1 & $\begin{array}{c}1 \\
6 . \\
6 \\
6\end{array}$ \\
\hline No contestó & 1 & $\begin{array}{r}2 . \\
44 \\
\end{array}$ & 1 & 2.86 & 0 & 0 \\
\hline \multicolumn{7}{|c|}{ Experiencia específica (años) } \\
\hline $1 \mathrm{a} 4$ & 5 & $\begin{array}{r}12 \\
.2 \\
0\end{array}$ & 3 & 8.57 & 2 & $\begin{array}{r}3 \\
3 . \\
3 \\
3\end{array}$ \\
\hline 5 a 8 & 11 & $\begin{array}{r}26 \\
.8 \\
0\end{array}$ & 10 & $\begin{array}{r}28.5 \\
7\end{array}$ & 1 & $\begin{array}{r}1 \\
6 . \\
6\end{array}$ \\
\hline 9 o más & 25 & $\begin{array}{r}61 \\
.0 \\
0\end{array}$ & 22 & $\begin{array}{r}62.8 \\
6\end{array}$ & 3 & $\begin{array}{r}5 \\
0 . \\
0 \\
0\end{array}$ \\
\hline
\end{tabular}

Fuente: Datos propios. $X=$ promedio. $\mathrm{DE}=$ desviación estándar. $\mathrm{N}=$ número.

La nacionalidad de los encuestados fue mayoritariamente del mismo país donde laboran, excepto por dos de EE.UU que trabajan en Puerto Rico, un búlgaro en Colombia, y un japonés y un venezolano en
Guatemala. Se refleja en este dato, que los procesos de planificación deportiva de los encuestados, esencialmente están direccionados y orientados por los entrenadores locales, rescatándose el esfuerzo de algunos países y deportes por contar con la presencia de entrenadores extranjeros, como una manera de reconocer y aprovechar las potencialidades que tienen otros países y por ende de contribuir a la universalización del deporte (Rodriguez Abreu, 2010).

En cuanto a la formación académica, entre los que tienen título universitario, dos son diferentes al área deportiva, así mismo entre los postgraduados hay cinco (un $22 \%$ ) en áreas completamente diferentes al deporte. Según los datos, los entrenadores encuestados en el nivel de selección nacional, tienen en su mayoría formación superior, lo cual puede contribuir en el desarrollo adecuado y efectivo en los procesos de planificación perspectiva del entrenamiento con influencia en lo físico, cognitivo, social y educativo (Acosta, 2012) (Capetillo, 2011) (Contreras, Rojas, \& Palomino, 2012) (Martens, 2002) (Navarro, 2004) de los deportistas escolares a su cargo. Además de lo anterior, se resalta el tiempo de experiencia como entrenadores deportivos de los entrenadores evaluados, lo cual permite suponer que sus procesos de planificación deportiva, están fundamentados en la formación profesional inicial de tercer ciclo (Posgrado), en la apropiación de los conocimientos y los saberes específicos, y en la experiencia adquirida (Salinero \& Ruiz, 2010), lo cual contribuye a elaborar una adecuada planificación en el desarrollo de los procesos de formación deportiva (Acosta, 2012) (Capetillo, 2011) (Contreras, Rojas, \& Palomino, 2012) (Saura, 1996).

Preguntados si seguían alguno de los modelos de planificación referidos en la literatura, $34(82.9 \%)$ contestaron que sí; 6 $(14.6 \%)$ que no y uno $(2.5 \%)$ no contestó. Los 
modelos seguidos por los entrenadores encuestados aparecen en la Tabla 2. Algunos de estos modelos no figuran en la literatura y pueden corresponder a combinaciones de métodos establecidos, a desconocimiento de la denominación "académica" de algunos de ellos o a verdaderas propuesta innovadoras de entrenadores del área.

Tabla 2. Frecuencia y porcentaje de los modelos de planificación utilizados por los entrenadores encuestados en función de grupos de deportes.
Fuente: Datos propios. Convenciones: Resist. = deportes de resistencia, Fza-vel.= deportes de fuerza velocidad, Pelota= deportes de pelota, Comb. $=$ deportes de combate, $P A C=$ deportes de precisión y arte competitivo, $N R=$ no responde, $A T R=$ acumulación, transformación, realización, $P D C=$ preparación directa a la competencia.

En relación al tiempo de planificación, se les interrogó si planificaban a largo, medio o corto plazo, si anual u otro o todas las anteriores. Las respuestas fueron que planificaban a largo plazo 18 casos (44.0\%), a mediano plazo $5(12.3 \%)$, anual $7(17.1 \%)$, todos los

\begin{tabular}{|c|c|c|c|c|c|c|}
\hline MODELO & $\begin{array}{l}\text { Todos } \\
(\%)\end{array}$ & Resist. & $\begin{array}{l}\text { Fza- } \\
\text { vel. }\end{array}$ & Pelota & Comb. & $\begin{array}{l}\text { PAC anteriores } 3(7.3 \%) \text {, largo plazo y anual } \\
(2.4 \%) \text { largo, medio plazo y anual } 3(7.3 \%\end{array}$ \\
\hline Macrociclos & $\begin{array}{r}10 \\
(37,04)\end{array}$ & - & $\begin{array}{r}6 \\
\text { (14.6) }\end{array}$ & $\begin{array}{r}3 \\
(7.3)\end{array}$ & - & $\begin{array}{l}\text { - largo1y mediano plazo } 2(4.8 \%) \text {, y finalment } \\
\text { |argơ), medio y corto plazo } 2(4.8 \%) \text {. }\end{array}$ \\
\hline ATR & $\begin{array}{r}4 \\
(14,81)\end{array}$ & $1(2.4)$ & - & $\begin{array}{r}1 \\
(2.4)\end{array}$ & $2(4.9)$ & $\begin{array}{l}\text { Se aprecia la diversidad en los modelos } \\
\text { planifidación del entrenamiento, lo cual }\end{array}$ \\
\hline Cubano & $\begin{array}{r}3 \\
(11.11)\end{array}$ & - & - & & $2(4.9)$ & $\begin{array}{l}\text { domprensible por la diversidad de deport } \\
\text { modalididades, condiciones laborales de } \\
\text { entrenadores (según lo manifestado por }\end{array}$ \\
\hline Anual & $\begin{array}{r}22 \\
(7.41)\end{array}$ & - & $\begin{array}{r}1 \\
(2.4)\end{array}$ & - & $1(2.4)$ & $\begin{array}{l}\text { - entreñadores, algunos fueron contrat } \\
\text { solamente para venir a Juegos, otros pc }\end{array}$ \\
\hline Programación & $\begin{array}{r}2 \\
(7.41)\end{array}$ & - & - & - & - & $\begin{array}{l}\text {-semestre, un año, otros están vincu } \\
\text { perthilanentemente y algunos incluso lo }\end{array}$ \\
\hline Tradicional & $\begin{array}{r}1 \\
(3.70)\end{array}$ & - & - & $\begin{array}{r}1 \\
\text { (2.4) }\end{array}$ & - & $\begin{array}{l}\text { - gd -honorem), calendarios esco } \\
\text { gondicilones climáticas o estacionarios }\end{array}$ \\
\hline Platonov & $\begin{array}{r}1 \\
(3.70)\end{array}$ & - & - & $\begin{array}{r}1 \\
(2.4)\end{array}$ & - & $\begin{array}{l}\text { región (Weineck, 2005) entre otros facto } \\
\text { que pueden afectar el proceso } \\
\text { preparación. Tampoco suele haber certeza }\end{array}$ \\
\hline$P D C$ & $\begin{array}{r}1 \\
(3.70)\end{array}$ & - & - & $\begin{array}{r}1 \\
\text { (2.4) }\end{array}$ & - & $\begin{array}{l}\text { - dontar con el equipo interdisciplinario, e } \\
\text { inseguridad de participación en los eve }\end{array}$ \\
\hline Personal & $\begin{array}{r}1 \\
(3.70)\end{array}$ & - & - & - & $1(2.4)$ & $\begin{array}{l}\text {-deportivos (Pazo, Sáenz-López, \& Frad } \\
\text { 2012), todo lo cual determina el no te }\end{array}$ \\
\hline Bloques & $\begin{array}{r}1 \\
(3.70)\end{array}$ & 1 (2.4) & - & - & - & del deporte de rendir \\
\hline $\begin{array}{l}\text { Esp. Tenis } \\
\text { Campo }\end{array}$ & - & - & - & $\begin{array}{r}1 \\
\text { (2.4) }\end{array}$ & - & $\begin{array}{l}\text { nificaciones perspectiv } \\
\text { eyev, 1977) (Vasconc }\end{array}$ \\
\hline$N R$ & $\begin{array}{r}14 \\
(34.1)\end{array}$ & - & $\begin{array}{r}2 \\
(4.9)\end{array}$ & $\begin{array}{r}5 \\
(12.2)\end{array}$ & $\begin{array}{r}6 \\
(14.6)\end{array}$ & en el perfeccionam \\
\hline
\end{tabular}
Con respecto a los criterios tenidos en cuenta para determinar el modelo de planificación a seguir, 2 (5\%) dijeron basarse en el 
calendario de competencias, $10(25 \%)$ en los objetivos para la competencia, $5(12.5 \%)$ en el calendario competitivo y los objetivos, $4(10 \%)$ en el calendario y los deportistas, $2(5 \%)$ tienen otros criterios y 17 (43.1\%) asumen todos los criterios anteriores.

Utilizando el estadígrafo Rho de Spearman, fueron encontradas relaciones entre variables cualitativas, así: entre edad y experiencia especifica (Rho $\left.=0.585^{* *}, \quad P=0.000\right)$, entre sexo y tipo de planificación empleada (Rho $=0.321^{*}, P=0.041$ ), entre sexo y contar con equipo interdisciplinario (Rho $=0.478^{* *}$, $\mathrm{P}=0.002$ ) y entre criterios de planificación y cuál modelo de planificación utiliza (Rho $=0.463^{*}, P=0.015$ ).

Respecto a las variables cuantitativas, mediante el coeficiente de correlación de Pearson, no fueron encontradas correlaciones significativas $(P>0.05)$.

\section{DISCUSIÓN}

La edad media de los entrenadores fue inferior a la encontrada en los Juegos Suramericanos Medellín 2010 (Ramos, Ayala, \& Aguirre, 2012) de 46.1 \pm 10.2 años, pero superior a la de los entrenadores españoles de triatlón de alto rendimiento (Ruiz \& Salinero, 2011) de $37.43 \pm 7.27$ años. Otro estudio (Feu, Ibáñez, \& Gozalo, 2010) reportó $33.41 \pm 8.74$ años como edad media de los 269 entrenadores españoles $(88.8 \%$ de ellos hombres) de balonmano escolar. Lo anterior muestra que la edad para actuar como entrenadores, presenta variabilidad, ya sea, por región geográfica, tipo de deporte y modalidad e incluso del evento deportivo en que se participa. Se rescata en estos entrenadores que se ubican en la edad de la madurez adulta, lo que posibilita tener ciertas características personales, sociales y formativas para desarrollar la planificación deportiva en procesos de formación, ya que los deportistas participantes se encuentran entre los 14 y 17 años de edad, siendo estas ideales para intervenir en todas sus dimensiones humanas (Pazo, Sáenz-López, \& Fradua, 2012) (Viciana \& Zabala, 2004), a partir de la misma experiencia activa, de su profesionalización y de la seguridad en el control de los procesos, desde la experiencia, la cordialidad y la responsabilidad (Pérez \& Pérez, 2009).

En cuanto a los modelos de planificación utilizados, los entrenadores centroamericanos dieron prelación a los macrociclos, seguidos del ATR y cubano, mientras la investigación de los entrenadores de Juegos Suramericanos 2010 (Ramos, Ayala, \& Aguirre, 2012), prefirieron en su orden los modelos tradicional, ciclos, bloques y ATR, por su parte en una comparación de modelos en entrenadores españoles (García-Pallares, García-Fernández, Sánchez-Medina, \& Izquiertdo, 2015) se concluyó que el modelo de bloques es más eficiente que el tradicional, claro está en deportistas de alto nivel. Estos resultados estuvieron acordes con un metaanálisis (Dantas, y otros, 2011) que identificó que el mejor modelo de planificación es el tradicional de Matveyev, seguido de los bloques de Verkhoshansky; tercero el de Bompa, cuarto el ATR, y por último las campanas estructurales de Forteza, mediante un estudio que abarcó 103 citaciones y elaboró la clasificación a través de un Índice de Adecuabilidad. Lo encontrado no coincide con estudios en categoría base (Moreno, 2004) (Unierzyski, 2005) (Verjoshansky Y. , 1990), para quienes hay un consenso, incluso entre los más severos críticos del modelo tradicional, de que este se constituye en el modelo más indicado para lograr el éxito a largo plazo, si bien existen modelos probablemente mejores, para cuando se trata de deportistas ya formados, lo cual no es el caso en los participantes en estos Juegos Escolares. 
Los entrenadores encuestados manifestaron preferir la planificación a largo plazo, lo cual es una de las pretensiones dentro del contexto del entrenamiento deportivo, ya que permite adecuados desarrollos en la preparación y formación del deportista escolar (Bompa, 2003) (Matveyev, 1977) (Matveyev, 2001) (Vasconcelos, 2005), dando respuesta a las actuales tendencias globales del entrenamiento deportivo, en el desarrollo adecuado de las diferentes capacidades y condiciones del mismo (Pérez \& Pérez, 2009) en tiempos prolongados de entrenamiento y en procura de altos logros.

\section{CONCLUSIONES}

El tiempo de planificación empleado por los entrenadores participantes en los Juegos Centroamericanos fue principalmente a largo y mediano plazo.

En cuanto al criterio para determinar el modelo de planificación a seguir, en primer lugar utilizan todos los criterios propuestos, seguido de los objetivos para la competencia, el calendario de competencias y la opinión de los deportistas.

La gran mayoría de entrenadores encuestados, manifiestan que tienen conocimiento y utilizan alguno de los modelos de planificación conocidos en la literatura, tales como Macrociclos, ATR, Cubano, Programación, Bloques, Personal, Macrociclo Alternado y Alternante, Semanal/anual, Modelo específico (caso tenis de campo), Anual, Campanas, Tradicional, Platonov y PDC. Casi uno de cada cinco de estos entrenadores no da cuenta del modelo utilizado.

\section{AGRADECIMIENTOS}

A la Vicerrectoría de Investigaciones y Postgrados de la Universidad de Caldas. A los estudiantes del programa de Licenciatura en Educación Física de la Universidad del Quindío colaboradores en la recolección de los datos.

\section{REFERENCIAS BIBLIOGRÁFICAS}

Acosta, E. (2012). Iniciación y formación deportiva: una reflexión siempre oportuna. (UDCA, Ed.) Actualidad y Divulgación Científica, 15(Suplemento olímpico), 57-65.

Ayala, C., Alzate, D., \& Aguirre, H. (diciembre de 2014). Preparación del deportista en el proceso de iniciación y formación deportiva en centroamérica. (U. d. Pamplona, Ed.) Actividad física y desarrollo humano., 6(1), 1-10.

Barbanti, J. (1979). Teoria e pratica do treinamento desportivo. Sao Paulo: Edgar Blucher.

Bompa, T. (2002). Periodizacao: Teoria e metodologia do treinamento. Porto Alegre: Porte.

Bompa, T. (2003). Teoría y metodología del entrenamiento deportivo. Madrid: Hispano Europea.

Campos, G., \& Cervera, V. (2003). Teoria e planejamento do treinamento desportivo. Sao Paulo, Brasil: Artmed.

Capetillo, R. (2011). Factores sociales que influyen en la formación deportiva de adolescentes. (U. Pedagógica, Ed.) Lúdica Pedagógica, 16(2), 39-48.

Contreras, D., Rojas, D., \& Palomino, O. (2012). Efectos de dos formas de periodizar la carga (lineal y doble ondulada) en el entrenamiento de la fuerza, sobre la cinemática de la salida estática voluntaria en patinadores de carreras pre púberes. (U. d. Pamplona, Ed.) Actividad Física y Desarrollo Humano, 4(1), 89-96.

Fernández, L. (1981). O treinamento desportivo. Procedimentos, organizacao e metodos. Sao Paulo: Editora Pedagogica Universitaria.

Filin, V. (1996). Desporte juvenil: teoria e metodologia. Sao Paulo: Centro de Informacoes Desportivas. 
García, J., \& Navarro, V. R. (1996). Bases teóricas del entrenamiento deportivo: Principios y aplicaciones. Barcelona: Gymnos.

García-Pallares, M., García-Fernández, L., Sánchez-Medina, S., \& Izquiertdo, M. (2015). Cambios en el rendimiento de piraguistas de nivel mundial tras la aplicacion de dos modelos diferentes de periodización del entrenamiwento. PuliCe Premium, 9(3), 1-10.

Gomes, A. (1998). Treinamento desportivo: processo de muitos anos de preparacao. Sao Paulo: Martins.

Gomes, A. (2009). Treinamento desportivo: estrutura e periodizacao. Sao Paulo: Artmed.

Gómez, P. (2011). El entrenamiento deportivo en el siglo XXI. Madrid: Alcalá.

Issurin, V. (2012). Entrenamiento deportivo. Peridización en bloques. Barcelona: Paidotribo.

Lanier, A. (1997). Introducción a la teoría y método del entrenamiento deportivo. Habana: Científico Técnica.

Machado, A. (2011). Bases metodologicas da preparacao fisica. Sao Paulo: Icone.

Martens, R. (2002). El entrenador de éxito. Barcelona: Paidotribo.

Martin, D., Nicolaus, J., Ostrowski, C., \& K., R. (2004). Metodología general del entrenamiento infantil y juvenil. Barcelona: Psidotribo.

Matveyev, L. (1977). Periodizacion del entrenamiento. Buenos Aires: INEF.

Matveyev, L. (1985). Fundamentos del entrenamiento deportivo. Moscú: Ráduga.

Murcia, N., Taborda, J., \& Ángel, L. (1998). Escuelas de formación deportiva y entrenamiento deportivo infantil: un enfoque integral. Armenia: Kinesis.

Navarro, F. (2004). Entrenamiento adaptado a los jóvenes. Revista de Educación(335), 6180.

Navarro, F., A, O., \& A., R. (2010). Planificación del entrenamiento y su control. . Madrid: Real Federación Española de Natación.

Pazo, C., Sánez-López, P., \& Fradua, L. (2012). Influencia del contexto deportivo en la formación de los futbolistas de la selección española de fútbol. Revista de Psicología del Deporte., 291-299.

Platonov, V. (2001). Teoría general del entrenamiento olímpico. Barcelona: Paidotribo.

Ramos, S., Ayala, C., \& Aguirre, H. (Mayo de 2012). Planificación del entrenamiento en Juegos Suramericanos Medellín 2010: modelos, duración y criterios. (U. UDCA, Ed.) UDCA Actualidad \& Divulgación Científica., 15(Suplemento especial), 6776.

Roberton, M., \& Halverson, L. (1984). Developing children- their changing movement: $A$ guide for teachers. Philadelphia, EUA: Lea \& Febiger.

Rodriguez Abreu, M. (Agosto de 2010). El origen del deporte contemporáneo en países centrales y su legado en la evolución de la educación física. EFdeportes, 15(147), 1-10.

Rusch, H., \& Weineck, J. (2004). Entrenamiento y práctica deportiva escolar. Barcelona: Paidotribo.

Salinero, J., \& Ruiz, G. (2010). El entrenador de alto rendimiento deportivo y su contraste con entrenadores de menor nivel. Estudio en la modalidad de karate-do en España. European Journal of Human Movement, 24(1), 107-119.

Saura, J. (1996). El entrenador en el deporte escolar. Lérida: Fundació Pública Institut d’Estudis Llerdencs.

Vasconcelos, A. (2005). Planificación y organización del entrenamiento deportivo. Barcelona: Paidotribo.

Verjoshansky, Y. (2001). Treinamento desportivo: Teoría y metodología. Sao Paulo, Brasil: Artmed.

Weineck, J. (2005). Entrenamiento total. Barcelona: Paidotribo. 